\title{
PREVALENCIA Y FACTORES ASOCIADOS AL CONSUMO DE CITRATO DE SILDENAFIL EN ESTUDIANTES UNIVERSITARIOS COLOMBIANOS, DURANTE EL PRIMER SEMESTRE DE 2013
}

\section{PREVALENCE AND ASSOCIATED FACTORS TO TADALAFIL CONSUMPTION IN COLOMBIAN COLLEGE STUDENTS DURING FIRST SEMESTER OF 2013}

\section{TITULO CORTO: PREVALENCIA Y FACTORES ASOCIADOS AL CONSUMO DE CITRATO DE SILDENAFIL}

Javier Martínez-Torres ${ }^{1}$, Ingrid Katherine Duarte-Corredor ${ }^{2}$, Gaby Johanna García-Vásquez ${ }^{3}$ y Gissely Dimarhy Jáuregui-Rodríguez ${ }^{4}$

Recibido en febrero 11 de 2015

Aceptado en mayo 12 de 2015

RESUMEN

El conocimiento de las prácticas sexuales en los adultos jóvenes es necesario para ejecutar acciones preventivas, enfocadas a la disminución de enfermedades de transmisión sexual y embarazos no deseados. Determinar la prevalencia del consumo de Citrato de Sildenafil y sus factores asociados en estudiantes universitarios, durante el primer semestre de 2013. Estudio descriptivo, desarrollado entre abril y junio del año 2013. El tamaño de la muestra fue de 340 estudiantes universitarios, todos masculinos, entre 18 y 26 años; toda la información fue recolectada por medio de una encuesta estructurada. La prevalencia del consumo de Viagra o Citrato de Sildenafil fue del 7,56\% (IC $95 \%$ 4,6\%-10,6\%); los principales factores predisponentes para su uso son: sufrir de disfunción eréctil (OR 14,72 IC95\% 5,29-40,96), al realizar el ajuste multivariado esta asociación aumenta 50\% (OR 21,67 IC95\% 6,27-74,89) y la curiosidad por el medicamento (OR 4,21 IC95\% 1,63-11,3). El consumo de Citrato de Sildenafil, aunque tiene una prevalencia baja, se relaciona, principalmente, con haber sufrido de episodios de disfunción eréctil, y la curiosidad por experimentar los efectos de ese medicamento.

Palabras clave: Adulto joven; conducta sexual; disfunción eréctil; conducta social (Fuente: DeCS)

\section{ABSTRACT}

The knowledge of sexual practices in young adults is required to run preventive actions aimed at the reduction of transmitted sexual diseases and unwanted pregnancies. To determine the prevalence Sildenafil Citrate

\footnotetext{
1. Especialista en Epidemiología; fisioterapeuta docente Universidad de Pamplona. Departamento de Enfermería. Grupo El Cuidar; Pamplona, Colombia. Correo: epid_javier@hotmail.com

2. Estudiante de Terapia Ocupacional, Universidad de Pamplona. Pamplona, Colombia Correo: ingridduarte01@hotmail.com

3. Estudiante de Terapia Ocupacional, Universidad de Pamplona. Pamplona, Colombia Correo: gabyjohanna77@gmail.com

4. Estudiante de Terapia Ocupacional, Universidad de Pamplona. Pamplona, Colombia Correo: chelymaria1@hotmail.com
} 
consumption and its associated factors in College Students, during the first half-year of 2013. A crosssectional, descriptive study was conducted between April and June of 2013, the sample size was 340, and all of them are male college students, aged 18 to 26 years; all information were collected using a structured survey. The prevalence of Viagra or Sildenafil Citrate was 7,56\% (CI95\% 4,6\%-10,6\%); the main predisposing factors for its use is to suffer of Male Erectile Dysfunction (OR 14.72, CI95\% 5.29 to 40.96), when it was adjusted by a multivariate model this association increases about 50\% (OR 21.67, CI95\% 6.27 to 74.89) and curiosity about this drug (OR 421 CI95\% 1.63 to 11.3) is the second one. Although Sildenafil Citrate consumption has low prevalence; it is mainly related with men who have suffered erectile dysfunction and curiosity to experience its effects.

Keywords: Young adult; sexual behavior; erectile dysfunction; social behavior (Fuente: MeSH).

\section{INTRODUCCION}

E Citrato de Sildenafil (CS), o Viagra, es uno de los fármacos utilizados en el tratamiento inicial de la disfunción eréctil masculina (DEM) ${ }^{1,2}$. Este medicamento también se usa en el tratamiento de la hipertensión pulmonar en adultos ${ }^{3,4}$, neonatos ${ }^{5}$ e infantes ${ }^{6}$. El medicamento fue aprobado en Estados Unidos por la Food Drugs Administration, el 27 de marzo del año 1998 ${ }^{7}$, y se le concedió la licencia para su venta en Europa por la Agencia Europea de Evaluación de Medicamentos, a mediados de septiembre del mismo año ${ }^{8}$. Aunque este medicamento fue, inicialmente, diseñado para el tratamiento de la hipertensión arterial sistémica y cardiopatía isquémica, en los primeros ensayos clínicos se encontró que tenía efectos en la erección peniana y en la respuesta sexual'; posteriormente, se describió que solo produce estos efectos entre personas de sexo masculino ${ }^{10}$. Para el caso de la DEM se indica, exclusivamente, como terapia farmacológica inicial; las propiedades que tiene el CS para intensificar y prolongar la respuesta eréctil del pene han hecho que se use de manera indiscriminada e "ilícita", pudiendo llegar a tener un alto potencial de abuso en términos de autodosificación a niveles o frecuencia inadecuadas ${ }^{11}$.

La DEM es un trastorno de la función sexual en los hombres definida como la incapacidad persistente de lograr o mantener la erección peniana suficiente para tener relaciones sexuales satisfactorias ${ }^{12}$. Eso conduce a que se produzcan unas alteraciones tales como: reducción en el deseo o libido, una disminución de la excitación, disminución en la frecuencia de las relaciones sexuales 0 incapacidad para alcanzar un orgasmo ${ }^{13}$.
Se ha demostrado una serie de efectos secundarios en las personas que toman CS, tales como bajas en la presión arterial ${ }^{11}$; dolor de cabeza, dispepsia, diarrea, congestión nasal, indigestión, alteraciones visuales, mareos y erupción ${ }^{14,15}$. Contar con un diagnóstico inicial del consumo de ese medicamento es fundamental para la detección temprana de las alteraciones y estrategias oportunas de intervención. Esto es de suma importancia, ya que se ha descrito que la automedicación y el uso indiscriminado de medicamentos es un problema de salud pública en adultos jóvenes ${ }^{16}$. Por tanto, el objetivo de este estudio fue determinar la prevalencia y los factores asociados del consumo de Citrato de Sildenafil en estudiantes universitarios de 18 a 25 años.

\section{MATERIALES Y MÉTODOS}

\section{Tipo y diseño de estudio}

Se realizó un estudio descriptivo transversal, cuya información se obtuvo por medio de un cuestionario autoadministrado y totalmente anónimo, en el cual se incluyó información acerca de variables sociodemográficas y de factores relacionados con consumo de CS. Los criterios de selección que se tuvieron en cuenta para incluir los sujetos en el estudio fueron: tener entre 18 años y 26 años, y ser de sexo masculino. El cuestionario se entregó después de la firma del consentimiento informado. Los criterios de inclusión fueron: ser mayor de 18 y menor de 26 años, firmar el consentimiento informado, aceptar ser parte de la investigación y encontrarse actualmente matriculado como estudiante en la universidad. Las personas que no habían iniciado 
relaciones sexuales fueron excluidas del análisis, no se tomó como criterio de exclusión el no haber iniciado relaciones sexuales, ya que las personas podían acusar haber tenido relaciones sexuales sin realmente haberlas tenido, lo que se convertiría en un sesgo por respuesta de presión social.

\section{Población y muestra}

Para el cálculo de la muestra se tomó la población total masculina matriculada para el primer semestre en la universidad, que fue de 3968 personas, con una frecuencia esperada del $7 \%{ }^{17}$, 18 ; un error aceptable del $3 \%$ y un intervalo de confianza de $95 \%$, lo que dio como resultado un total de 260 personas. Este resultado se aumentó en un $30 \%$ por posible no respuesta, y quedó como total una muestra de 340 personas.

\section{Factores sociodemográficos y de comportamientos}

Para el presente análisis se definieron como factores asociados las siguientes variables sociodemográficas: 1.) Edad (menor de 19,9, entre 20 y 22,9 y mayor de 23 años); 2.) semestre (hasta tercer semestre, entre cuarto y sexto semestres, y séptimo semestre o superior); 3.) edad de inicio de relaciones sexuales (antes de 14 años, entre 15 y 18 años, y después de los 19 años; 4.) uso del condón (sí y no); 5.) conoce los efectos del CS (sí y no); 6.) haber sufrido de DEM (sí y no); 7.) asesoría para el uso del CS (sí y no); 8.) asesoría para el uso del CS Por parte de un farmaceuta (sí y no).

\section{Evaluación del consumo de Citrato de Sildenafil y DEM}

La valoración de la DEM se tomó como el hecho de haber tenido incapacidad para mantener una erección en los últimos doce (12) meses: mientras que el consumo del CS se evaluó como el consumo del medicamento en los últimos doce (12) meses.

\section{Análisis estadístico}

Se inició con la exclusión de las personas que no habían iniciado vida sexual activa, las cuales fueron eliminadas de la base de datos por medio de una pregunta que decía: ¿Ha tenido relaciones sexuales alguna vez en su vida? De igual manera también fueron excluidas las personas que no respondían alguna de las variables que se estudiaron como factores asociados. Posterior a la depuración de los datos se realizó un análisis exploratorio para determinar la distribución de frecuencias (medidas de tendencia central y de dispersión para variables cuantitativas) y frecuencias relativas (para variables cualitativas). Para estimar la relación entre el consumo de CS y las variables sociodemográficas de los estudiantes, se utilizaron cuatro modelos de regresión logística binaria. El primer modelo de regresión logística binaria fue simple (OR no ajustado), el segundo modelo fue ajustado por edad, semestre y edad de inicio de relaciones sexuales (modelo $\mathbf{1}^{\text {a }} \mathbf{O R}$ ). Los análisis fueron realizados en SPSS versión 20 y se consideró como significativo un valor $P<0,05$.

\section{Consideraciones éticas}

El estudio se desarrolló de acuerdo con las normas establecidas en la Resolución 8430 de 1993 del Ministerio de Salud. De acuerdo con su artículo 11 este estudio se clasifica como una investigación "sin riesgo", ya que no se realizó ninguna intervención o modificación intencionada de las variables biológicas, fisiológicas, sicológicas o sociales de los individuos que participan en el estudio. La participación fue voluntaria y quienes aceptaron formar parte del estudio firmaron un consentimiento informado. Los datos fueron manejados con estricta confidencialidad y anonimato, sin realizarse análisis individuales; el formato de valoración estaba numerado con un código el cual solamente fue útil para el proceso de tabulación, y estaba separado del registro de consentimiento informado.

\section{RESULTADOS}

La muestra estuvo constituida por 340 estudiantes universitarios, de los cuales se analizaron datos de 307, quienes contestaron completamente el cuestionario, o quienes habían iniciado vida sexual en el momento de la realización del estudio. La edad media de los estudiantes que de 21,02 años DE 2,35. La edad media de inicio de relaciones sexuales fue a los 15,29 años DE 2,21 años. Aproximadamente, el 50\% de los encuestados se encontraban cursando los primeros tres semestres, mientras que entre el cuarto y el sexto semestres estaba compuesto por un $29 \%$, los restantes $21 \%$ estaban en los últimos semestres. La prevalencia del consumo de CS fue de 7,56\% (IC 95\% 4,6\%-10,6\%); los individuos que acusaron haber sufrido de DEM, los que iniciaron relaciones sexuales después de los 19 años y estar en séptimo o semestre superior a los semestres del séptimo o superior, mostraron las mayores prevalencias $(45,0 \%$, $14,29 \%$ y $14,00 \%$ ), respectivamente. Los demás datos se muestran en la tabla 1. 
Tabla 1. Distribución de los Factores Asociados para consumo de Citrato de Sildenafil, en estudiantes universitarios durante el primer semestre de 2013

\begin{tabular}{|c|c|c|c|c|}
\hline & \multicolumn{2}{|c|}{ Sí } & \multicolumn{2}{|c|}{ NO } \\
\hline & Número & Porcentaje & Numero & Porcentaje \\
\hline Total & 23 & 7,56 & 281 & 92,44 \\
\hline \multicolumn{5}{|l|}{ Edad } \\
\hline Menor de 19,9 & 6 & 6,32 & 89 & 93,68 \\
\hline Entre 20 y 22,9 & 8 & 6,67 & 112 & 93,33 \\
\hline Mayor de 23 & 9 & 10,11 & 80 & 89,89 \\
\hline \multicolumn{5}{|l|}{ Semestre } \\
\hline Hasta tercer semestre & 11 & 6,92 & 148 & 93,08 \\
\hline Entre cuarto y sexto semestre & 4 & 4,65 & 82 & 95,35 \\
\hline Séptimo semestre o superior & 7 & 14,00 & 43 & 86,00 \\
\hline \multicolumn{5}{|c|}{ Edad de inicio de relaciones sexuales } \\
\hline Antes de 14 años & 7 & 6,54 & 100 & 93,46 \\
\hline Entre 15 y 18 años & 14 & 7,82 & 165 & 92,18 \\
\hline Después de los 19 años & 3 & 14,29 & 18 & 85,71 \\
\hline \multicolumn{5}{|c|}{ Uso del condón en todas la relaciones } \\
\hline Sí & 19 & 7,85 & 223 & 92,15 \\
\hline No & 1 & 2,50 & 39 & 97,50 \\
\hline \multicolumn{5}{|l|}{ Conoce los efectos del CS } \\
\hline Sí & 14 & 10,94 & 114 & 89,06 \\
\hline No & 10 & 5,68 & 166 & 94,32 \\
\hline \multicolumn{5}{|l|}{ Haber sufrido de DEM } \\
\hline Sí & 9 & 45,00 & 11 & 55,00 \\
\hline No & 15 & 5,26 & 270 & 94,74 \\
\hline \multicolumn{5}{|l|}{ Asesoría para el uso del CS } \\
\hline Sí & 4 & 10,26 & 35 & 89,74 \\
\hline No & 18 & 6,90 & 243 & 93,10 \\
\hline \multicolumn{5}{|c|}{ Asesoría para el uso del CS Por parte de un farmaceuta } \\
\hline Sí & 3 & 10,34 & 26 & 89,66 \\
\hline No & 15 & 6,73 & 208 & 93,27 \\
\hline
\end{tabular}

Fuente: Elaboración propia

El análisis de regresión logística simple y múltiple, el cual muestra que conocer los efectos de CS (OR 2,59 IC95\% 1,01-6,43); haber sufrido de DEM (OR 21,67 IC95\% 6,27-
74,89) mostraron asociación con el consumo de CS. Los demás datos se muestran en las tablas 2 y 3. 
Tabla 2. Asociación de las variables sociodemográficas para consumo de Citrato de Sildenafil, en estudiantes universitarios durante el primer semestre de 2013

\begin{tabular}{|l|c|c|}
\hline EDAD $^{1}$ & OR & IC95\% \\
\hline Entre 20 y 22,9 & 1,06 & $0,35-4,17$ \\
\hline Mayor de 23 & 1,67 & $0,57-4,86$ \\
\hline SEMESTRE $^{2}$ & 1,52 & $0,47-4,94$ \\
\hline Hasta tercer semestre & 3,34 & $0,93-12,04$ \\
\hline Séptimo semestre o superior & \multicolumn{2}{|l|}{} \\
\hline EDAD DE INICIO $^{3}$ & 1,21 & $0,47-3,11$ \\
\hline Entre 15 y 18 años & 2,38 & $0,56-10,08$ \\
\hline Después de los 19 años &
\end{tabular}

Grupo de Referencia:

1. Menor de 19 años de edad

2. Entre cuarto y sexto semestre

3. Antes los 14 años.

Fuente: Elaboración propia

Tabla 3. Asociación de los factores estudiados para consumo de citrato de sildenafil, en estudiantes universitarios en durante el primer semestre de 2013

\begin{tabular}{|l|c|c|c|c|}
\hline Uso del condón ${ }^{1} \cdot$ & OR & IC95\% & ORA ${ }^{*}$ & IC95\% \\
\hline Sí & 3,32 & $0,43-25,41$ & 3,1 & $0,39-24,5$ \\
\hline Conoce los efectos del CS ${ }^{1}$. & 2,03 & $0,87-4,75$ & $\mathbf{2 , 5 9}$ & $\mathbf{1 , 0 1 - \mathbf { 6 , 4 3 }}$ \\
\hline Sí & 14,72 & $5,29-40,96$ & 21,67 & $6,27-74,89$ \\
\hline Haber sufrido de DEM & 1,54 & $0,49-4,82$ & 1,63 & $0,49-3,28$ \\
\hline Sí & & 1,60 & $0,39-3,52$ \\
\hline Asesoría para el uso del CS ${ }^{1}$.
\end{tabular}

1. Respuesta Negativa.

* Ajustado por Edad, Semestre y Edad inicio de relaciones sexuales.

Fuente: Elaboración propia

\section{DISCUSIÓN}

El principal hallazgo del presente estudio fue que el $7,53 \%$ de estudiantes de una universidad colombiana han consumido Citrato de Sildenafil. Aunque no hay muchos estudios que hubiesen analizado la prevalencia del consumo de CS en adultos jóvenes, se encontraron dos investigaciones americanas: la primera, realizada entre 234 hombres sexualmente activos en edades oscilantes entre 18 y 25 años, mostró que alrededor del $6 \%$ de los sujetos habían tomado $\mathrm{CS}^{17}$, similares resultados encontró Mansergh et al., en 388 hombres homosexuales ${ }^{18}$. 
El período de transición entre el final de la adolescencia y la adultez ha sido identificado como una etapa crítica en el desarrollo de los adultos jóvenes, está caracterizada por un continuo cambio social y sexual ${ }^{19}$. Los hombres jóvenes experimentan una serie de vivencias de alto riesgo, tales como experiencias sexuales, en las cuales debido al mercado actual se infunde la necesidad de mostrar una virilidad basada en la capacidad de responder en el acto sexual, que puede conducir al consumo de $\mathrm{CS}^{20}$. El principal factor predisponente para el consumo de CS es haber sufrido de DEM (OR 21,67 IC95\% 6,27-74,89), evento que afecta a los hombres en todos los momentos de la vida. Se describe que dos de cada tres hombres mayores de 40 años ${ }^{21}$, y alrededor de un $13 \%$ de los hombres entre 18 y 25 años, han sufrido este problema ${ }^{17}$.

Los farmaceutas pertenecen al sistema médico empírico, muchas veces realizan asesorías para recetar los medicamentos a sus usuarios ${ }^{22}$. En el presente estudio no se encontró asociación entre el recibir asesorías por parte del farmaceuta y el consumo de este medicamento (OR 1,60 IC95\% 0,49-3,28). Cabe resaltar que, aunque este medicamento está indicado para la DEM, se debe usar siempre por recomendación médica² ${ }^{2}$ Por la naturaleza de la sexualidad humana, siempre se buscará la satisfacción propia y la de la pareja, lo que llevará a la búsqueda de una serie de nuevas estrategias para cumplir esas metas; es en ese punto donde aparece la oportunidad de probar nuevas cosas por curiosidad, lo cual se ha demostrado como el causante del inicio en muchas adicciones, por ejemplo la del cigarrillo ${ }^{23}$. Lo anteriormente expuesto, sumado a la facilidad de adquisición que tiene este medicamento, pues se puede conseguir en cualquier droguería, sin necesidad de tener una fórmula médica, son mecanismos que facilitan su consumo. No se encontraron más estudios que pudieran contrastar los resultados del presente trabajo, ya que el consumo de CS es un fenómeno relativamente joven. El presente estudio tiene las limitaciones propias de los estudios transversales, que no permiten establecer causalidad entre las variables estudiados ${ }^{24}$.

A manera de conclusiones, el consumo de Citrato de Sildenafil, aunque tiene una prevalencia baja $(7,56 \%)$, se relaciona principalmente con haber sufrido de episodios de DEM. El consumo de este medicamento debe seguir estudiándose entre esa población para hacer intervenciones encaminadas a una sexualidad responsable, y prevenir la automedicación para la solución de problemas de tipo sexual. Es probable que la prevalencia del consumo de CS sea más alta, ya que por considerarse un tema tabú en la sociedad actual, el hombre que tenga DEM es tachado de perder su masculinidad. Lo anteriormente expuesto puede llevar a que se produzca un sesgo en la ponderación de la prevalencia real del consumo de CS.

\section{AGRADECIMIENTOS}

Los agradecimientos son para el Programa de Terapia Ocupacional y Enfermería, de la Universidad de Pamplona, por el apoyo brindado para el desarrollo de la presente investigación.

\section{CONFLICTO DE INTERESES}

Los autores declaran libremente no tener conflicto de intereses.

\section{REFERENCIAS BIBLIOGRÁFICAS}

1. Sairam K, Kulinskaya E, Hanbury D, Boustead G, McNicholas T. Oral sildenafil (Viagra ${ }^{\mathrm{TM}}$ ) in male erectile dysfunction: use, efficacy and safety profile in an unselected cohort presenting to a British district general hospital. BMC Urol. 2002; 18(2): 4-10.

2. Virag R. Indications and early results of sildenafil (viagra) in erectile dysfunction. Urology. 1999; 54(6): 1073-77.

3. Berger A, Edelsberg J, Teal S, Mychaskiw MA, Oster G. Changes in healthcare utilization and costs associated with sildenafil therapy for pulmonary arterial hypertension: a retrospective cohort study. BMC Pulm Med. 2012; 12(1): 75-82.

4. Preston I, Klinger JR, Houtches J, Nelson D, Farber HW, Hill NS. Acute and chronic effects of sildenafil in patients with pulmonary arterial hypertension. Resp Med. 2005; 99(12):1501-10.

5. Erickson S, Reyes J, Bohn D, Adatia I. Sildenafil (Viagra) in childhood and neonatal pulmonary hypertension. $\mathrm{J}$ Am Coll Cardiol. 2002; 39(2): 402-3.

6. Senior K. Viagra eases symptoms of pulmonary arterial hypertension in children. Drug Discov Today. 2005; 10 (20): 1337-1344.

7. Bradbury J. Is the honeymoon over for viagra (News). Lancet. 1998; 351(9116): 1635-42.

8. Brooks A. Viagra is licensed in Europe but rationed in Britain. BMJ. 1998; 317(9): 765-6.

9. Rosas M, Campos R, Morales JE. Sildenafil e inhibidores de fosfodiesterasa en hipertensión pulmonar. Neumol Cir Torax. 2006; 65(4): 186-200.

10. Nurnberg HG, Hensley PL, Lauriello J, Parker LM, Keith SJ. Sildenafil for women patients with antidepressantinduced sexual dysfunction. Psychiatr Serv. 1999; 50(8): 1076-78. 
11. Marmor MF, Kessler R. Sildenafil (Viagra) and Ophthalmology. Surv Ophthalmol. 1999; 44(2): 153-7.

12. NIH Consensus Conference Impotence. NIH Consensus Development Panel on Impotence. JAMA. 1993; 270(1): 83-90.

13. Kennedy SH, Rizvi S. Sexual dysfunction, depression, and the impact of antidepressants. J Clin Psychopharmacol. 2009; 29(2): 157-64.

14. Boolell M, Gepi-Attee S, Gingell JC, Allen MJ. Sildenafil, a novel effective oral therapy for male erectile dysfunction. British Jour Urol. 1996; 78(2): 257-261.

15. Goldstein I, Goldstein I, Lue TF, Padma-Nathan H, Rosen RC, Steers WD, Wicker PA. Oral sildenafil in the treatment of erectile dysfunction. N Engl J Med. 1998; 338(20): 1397-404.

16. Corrêa da Silva MG, Flores-Soares MC, Muccillo-Baisch AL. Self-medication in university students from the city of Rio Grande, Brazil. BMC Public Health. 2012; 12(5):339-46

17. Musacchio NS, Hartrich M, Garofalo R. Erectile Dysfunction and Viagra Use: What's up with College-Age Males? J Adolesc Health. 2006; 39(3): 452-54.

18. Mansergh G, Shouse RL, Marks G, Guzman R, Rader, M, Buchbinder $S$, et al. Methamphetamine and sildenafil (Viagra) use are linked to unprotected receptive and insertive anal sex, respectively, in a sample of men who have sex with men. Sex Transm Infect. 2006; 82(2): 131-4.

19. Furstenberg FF. The sociology of adolescence and youth in the 1990s: A critical commentary. J Marriage Fam. 2000; 62(4): 896-910.

20. Bynner J. Rethinking the youth phase of the life-course: The case for emerging adulthood? J Youth Stud. 2005; 8(4): $367-84$.

21. Grover SA, Lowensteyn I, Kaouache M, Marchand S, Coupal L, DeCarolis E, et al. The prevalence of erectile dysfunction in the primary care setting: importance of risk factors for diabetes and vascular disease. Arch Intern Med. 2006; 166(2): 213-9.

22. Guerrero R, Gonzalez CL, Medina E. Epidemiología. Santiago de Chile, Editorial Iberoamericana; 1986.

23. U.S. Department of Health and Human Services. Preventing Tobacco Use Among Youth and Young Adults: A Report of the Surgeon General. Atlanta, GA: U.S. Department of Health and Human Services, Centers for Disease Control and Prevention, National Center for Chronic Disease Prevention and Health Promotion, Office on Smoking and Health, 2012.

24. Hernández-Ávila M. Epidemiología: diseño y análisis de estudios. México, D.F. Editorial Médica Panamericana; 2007.

Para citar este artículo: Martínez-Torres J, Duarte-Corredor IK, García-Vásquez GJ, Jáuregui-Rodríguez GD. Prevalencia y factores asociados al consumo de citrato de sildenafil en estudiantes universitarios colombianos, durante el primer semestre de 2013. Duazary. 2015 dic; 12 (2): 118 - 124 